\title{
Polycrystalline thin-film transistors fabricated on high-mobility solid-phase- crystallized Ge on glass
}

Cite as: Appl. Phys. Lett. 114, 212107 (2019); https://doi.org/10.1063/1.5093952

Submitted: 26 February 2019 . Accepted: 12 May 2019. Published Online: 31 May 2019

K. Moto (D), K. Yamamoto (D), T. Imajo, T. Suemasu, H. Nakashima, and K. Toko

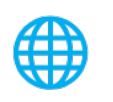

\section{ARTICLES YOU MAY BE INTERESTED IN}

Enhanced $n$-type $\beta-\mathrm{Ca}_{2} \mathrm{O}_{3}$ (201) gate stack performance using $\mathrm{Al}_{2} \mathrm{O}_{3} / \mathrm{SiO}_{2}$ bi-layer dielectric Applied Physics Letters 114, 212106 (2019); https://doi.org/10.1063/1.5089627

Atomic layer deposition of energy band tunable tin germanium oxide electron transport layer for the SnS-based solar cells with $400 \mathrm{mV}$ open-circuit voltage

Applied Physics Letters 114, 213901 (2019); https://doi.org/10.1063/1.5098766

Magnetically and optically tunable terahertz radiation from $\mathrm{Ta} / \mathrm{NiFe} / \mathrm{Pt}$ spintronic nanolayers generated by femtosecond laser pulses

Applied Physics Letters 114, 212405 (2019); https://doi.org/10.1063/1.5099201

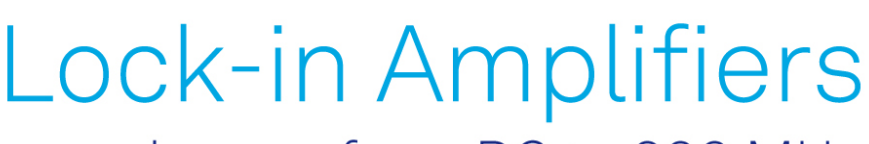

... and more, from DC to $600 \mathrm{MHz}$

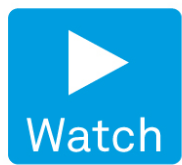




\title{
Polycrystalline thin-film transistors fabricated on high-mobility solid-phase-crystallized Ge on glass
}

\author{
Cite as: Appl. Phys. Lett. 114, 212107 (2019); doi: 10.1063/1.5093952 \\ Submitted: 26 February 2019 • Accepted: 12 May 2019 . \\ Published Online: 31 May 2019
}

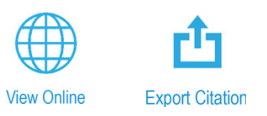

K. Moto, ${ }^{1,2}$ (D K. Yamamoto,, ${ }^{3, a)}$ (D T. Imajo, ${ }^{7}$ T. Suemasu, ${ }^{7}$ H. Nakashima, ${ }^{4}$ and K. Toko ${ }^{1,5, a)}$

\author{
AFFILIATIONS \\ IInstitute of Applied Physics, University of Tsukuba, 1-1-1 Tennodai, Tsukuba, Ibaraki 305-8573, Japan \\ 2JSPS Research Fellow, 8 Ichiban-cho, Chiyoda-ku, Tokyo 102-8472, Japan \\ ${ }^{3}$ Interdisciplinary Graduate School of Engineering Science, Kyushu University, Kasuga, Fukuoka 816-8580, Japan \\ ${ }^{4}$ Global Innovation Center, Kyushu University, Kasuga, Fukuoka 816-8580, Japan \\ ${ }^{5}$ PRESTO, Japan Science and Technology Agency, 4-1-8 Honcho, Kawaguchi, Saitama 332-0012, Japan
}

${ }^{a)}$ Authors to whom correspondence should be addressed: yamamoto.keisuke.380@m.kyushu-u.ac.jp and toko@bk.tsukuba.ac.jp

\begin{abstract}
Low-temperature formation of Ge thin-film transistors (TFTs) on insulators has been widely investigated to improve the performance of Si large-scale integrated circuits and mobile terminals. Here, we studied the relationship between the electrical properties of polycrystalline Ge and its TFT performance using high-mobility Ge formed on glass using our recently developed solid-phase crystallization technique. The field-effect mobility $\mu_{\mathrm{FE}}$ and on/off currents of the accumulation-mode TFTs directly reflected the Hall hole mobility $\mu_{\mathrm{Hall}}$, hole concentration, and film thickness of Ge. By thinning the 100 -nm thick Ge layer with a large grain size $(3.7 \mu \mathrm{m})$, we achieved a high $\mu_{\mathrm{Hall}}\left(190 \mathrm{~cm}^{2} / \mathrm{Vs}\right)$ in a 55-nm thick film that was almost thin enough to fully deplete the channel. The TFT using this Ge layer exhibited both high $\mu_{\mathrm{FE}}$ $\left(170 \mathrm{~cm}^{2} / \mathrm{Vs}\right)$ and on/off current ratios $\left(\sim 10^{2}\right)$. This is the highest $\mu_{\mathrm{FE}}$ among low-temperature $\left(<500^{\circ} \mathrm{C}\right)$ polycrystalline Ge TFTs without minimizing the channel region $(<1 \mu \mathrm{m})$.
\end{abstract}

Published under license by AIP Publishing. https://doi.org/10.1063/1.5093952

Ge has attracted attention as the most promising candidate for post-Si materials because it has a higher carrier mobility than $\mathrm{Si}$ and is compatible with the conventional Si process. Ge metaloxide-semiconductor field-effect transistor (MOSFET) mobilities have exceeded those of Si-MOSFETs because of the development of device technologies including gate stacks. ${ }^{1-6}$ However, leakage current in bulk $\mathrm{Ge}$ is inevitable because of the narrow bandgap $(0.66 \mathrm{eV})$. Ge-on-insulator (GOI) technology is the most promising solution. High quality GOI structures have been obtained by mechanical transfer, ${ }^{7-9}$ oxidationinduced condensation, ${ }^{5,10}$ rapid-melting growth, ${ }^{11-14}$ and lamp annealing. ${ }^{15,16}$ The GOI structure formed by these methods effectively suppresses the leakage current and improves the device performance of Ge-MOSFETs. These results demonstrate the potential of Ge transistors; however, their application is limited because these methods require a single-crystal wafer or high temperature process $\left(>500^{\circ} \mathrm{C}\right)$.

Research on low-temperature synthesis of polycrystalline Ge (poly-Ge) thin films on insulators has been conducted for decades. This enables monolithic integration of high-performance Ge thin-film transistors (TFTs) into Si-large-scale integrated circuits (LSIs) and flat panel displays. Ge-TFTs have been established using poly-Ge formed by solid-phase crystallization (SPC), ${ }^{17-20}$ laser annealing, ${ }^{21}$ seed layer technique, ${ }^{22}$ and metal-induced crystallization. ${ }^{23-27}$ Poly-Ge generally has a high hole concentration $p$ because of defect-induced acceptors and low Hall effect hole mobility $\mu_{\text {Hall }}$ due to grain boundary scattering. ${ }^{18,28}$ Although the miniaturization of the channel region $(<1 \mu \mathrm{m})$ and the multigate structure have successfully reduced the leakage current and improved MOSFET mobility, the crystallinity of poly-Ge is insufficient to overcome the performance of Ge-TFTs over Si-MOSFETs. To improve the performance of Ge-TFTs, it is essential not only to enhance the crystallinity of poly-Ge but also to comprehensively study the relationship between its electrical properties and TFT characteristics.

SPC progresses in two steps: nucleation and subsequent lateral growth. Because the grain size is determined by the balance between the nucleation frequency and the lateral growth rate, there is a possibility of enlarging the grain size by devising growth conditions. ${ }^{18} \mathrm{We}$ recently found that the densification of the amorphous $\mathrm{Ge}(\mathrm{a}-\mathrm{Ge})$ precursor significantly enlarged the grain size of poly-Ge due to lateral 
growth promotion in SPC. ${ }^{29}$ Moreover, the precursor densification reduced the trap-state density $\left(4.4 \times 10^{11} \mathrm{~cm}^{-2}\right)$ and energy barrier height $(6.4 \mathrm{meV})$ of the grain boundary, resulting in a $\mu_{\mathrm{Hall}}$ of $340 \mathrm{~cm}^{2} / \mathrm{Vs}^{29}$ Additionally, $\mu_{\text {Hall }}$ was updated to $620 \mathrm{~cm}^{2} / \mathrm{Vs}$ by thickening a-Ge, postannealing at $500^{\circ} \mathrm{C}$, and inserting $\mathrm{GeO}_{2}$ underlayer. ${ }^{30,31}$ This $\mu_{\text {Hall }}$ is the highest ever recorded for a thin film directly grown on an insulator at temperatures below $900^{\circ} \mathrm{C}$. In the present study, we fabricate poly-Ge TFTs using SPC-Ge and discuss the relationship between the film properties (thickness, $\mu_{\text {Hall, }}$ and $p$ ) and TFT characteristics (field-effect mobility: $\mu_{\mathrm{FE}}$ and on/off currents). We demonstrate the highest $\mu_{\mathrm{FE}}$ among low-temperature $\left(<500^{\circ} \mathrm{C}\right)$ polyGe TFTs without minimizing the channel region.

The a-Ge layers were deposited on $\mathrm{SiO}_{2}$ glass substrates using the Knudsen cell of a molecular beam deposition system (base pressure: $5 \times 10^{-7} \mathrm{~Pa}$ ). The initial film thickness of the a-Ge layer, $t_{\mathrm{i}}$, ranged from 25 to $200 \mathrm{~nm}$. The Ge layers were densified by heating the substrate at $125^{\circ} \mathrm{C}$ during deposition. ${ }^{29}$ The samples were then loaded into a conventional tube furnace in an $\mathrm{N}_{2}$ atmosphere and annealed at $450{ }^{\circ} \mathrm{C}$ for $5 \mathrm{~h}$ to induce SPC. Figures 1 (a) -1 (e) show the crystal orientation maps obtained by electron backscattering diffraction (EBSD). The EBSD images indicate that the grain size dramatically varies with $t_{\mathrm{i}}$, while the crystal orientation is almost random for all $t_{\mathrm{i}}$. Figure $1(\mathrm{f})$ shows that the highest grain size is obtained at $t_{\mathrm{i}}=100 \mathrm{~nm}$. This behavior likely reflects the balance of the precursor density and the stress in the Ge film. The heating deposition densifies the precursor at $t_{\mathrm{i}}>50 \mathrm{~nm}$, while the precursor density remains low at $t_{\mathrm{i}} \leq 50 \mathrm{~nm} .^{29,30}$ For $t_{\mathrm{i}}>50 \mathrm{~nm}$ where the precursor is densified, the grain size increases due to the lateral growth promotion. Conversely, in SPC, heterogeneous nucleation occurs at the film surface or substrate interface. The thin films have stress due to the difference in thermal expansion coefficients with the substrates, which retard the nucleation. ${ }^{32}$ The thicker film makes the surface stress more relaxed and then makes
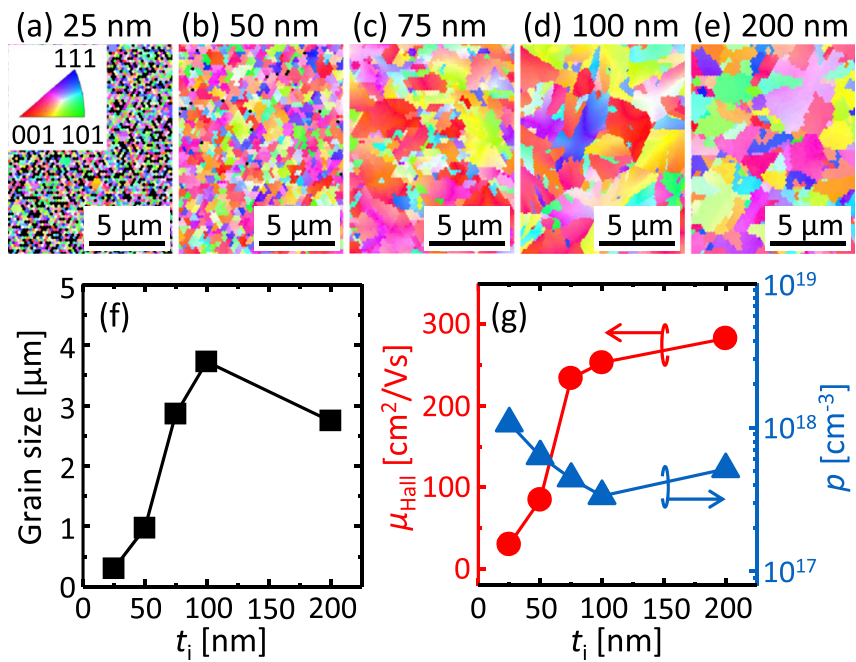

FIG. 1. Initial film thickness $t$ dependence of the grain size and electrical properties of the SPC-Ge layers. (a)-(e) EBSD images with various $t_{\mathrm{i}}$ values $(25,50,75,100$, and $200 \mathrm{~nm}$ ). The colors indicate the crystal orientation, according to the inserted color key. (f) Average grain size determined by EBSD analyses. (g) Hall hole mobility $\mu_{\text {Hall }}$ and hole concentration $p$ obtained by the Hall effect measurement with the van der Pauw method as a function of $t_{\mathrm{i}}$. surface nucleation more likely to occur, resulting in a smaller grain size. Reflecting the balance, the grain size is maximized at $t_{\mathrm{i}}=100 \mathrm{~nm}$. We used Hall effect measurements to evaluate the electrical properties of the SPC-Ge layers. All samples showed p-type conduction because the defects in Ge provide shallow acceptor levels that generate holes at room temperature. ${ }^{28}$ Therefore, larger grain sizes, that is, fewer grain boundaries, provide lower $p$, as shown in Figs. $1(\mathrm{f})$ and $1(\mathrm{~g})$. Figure $1(\mathrm{~g})$ also shows that $\mu_{\text {Hall }}$ peaks at $t_{\mathrm{i}}=200 \mathrm{~nm}$, whereas the grain size peaks at $t_{\mathrm{i}}=100 \mathrm{~nm}$. This behavior is likely attributed to the carrier scattering near the $\mathrm{Ge} / \mathrm{SiO}_{2}$ interface. ${ }^{30}$ Because the interface scattering is weaker for the thicker film, $t_{\mathrm{i}}=200 \mathrm{~nm}$ exhibits higher $\mu_{\text {Hall }}$ than $t_{\mathrm{i}}=100 \mathrm{~nm}$.

We fabricated accumulation-mode metal source/drain (S/D) p-channel TFTs using SPC-Ge with different $t_{\mathrm{i}}$ values (Fig. 1). Figure 2 shows the process and structure. First, SPC-Ge on glass was isolated into a $55-\times 155-\mu \mathrm{m}^{2}$ rectangle using a diluted $\mathrm{H}_{2} \mathrm{O}_{2}$ solution. ${ }^{2}$ Then, sacrificial oxidation was performed by electron cyclotron resonance (ECR) plasma oxidation forming $\mathrm{GeO}_{2}$ ( $1 \mathrm{~nm}$ thick). After removing the sacrificial oxide by wet treatment, photoresist was coated and patterned into S/D shape rectangles on both sides of the isolated Ge island. Pt and TiN (each $10 \mathrm{~nm}$ thick) as metal S/Ds were sequentially deposited using radio-frequency magnetron sputtering and patterned by removal of photoresist. Postmetallization annealing (PMA) was performed at $400^{\circ} \mathrm{C}$ for $30 \mathrm{~min}$ in $\mathrm{N}_{2}$ to form PtGe/Ge contacts. The $\mathrm{PtGe} / \mathrm{Ge}$ contacts have a low hole barrier height and are suitable for accumulation-mode p-channel Ge-TFTs. ${ }^{33}$ We used $\mathrm{Al} / \mathrm{SiO}_{2} / \mathrm{Al}_{2} \mathrm{O}_{3} /$ $\mathrm{SiO}_{2} / \mathrm{GeO}_{2}$ for the gate stack. $\mathrm{GeO}_{2}$ ( $3 \mathrm{~nm}$ thick) was formed by ECR plasma oxidation at $130^{\circ} \mathrm{C}$ and protected with an $\mathrm{SiO}_{2}$ film $(2 \mathrm{~nm}$ thick). $\mathrm{Al}_{2} \mathrm{O}_{3}$ (20 nm thick) was then deposited using atomic layer

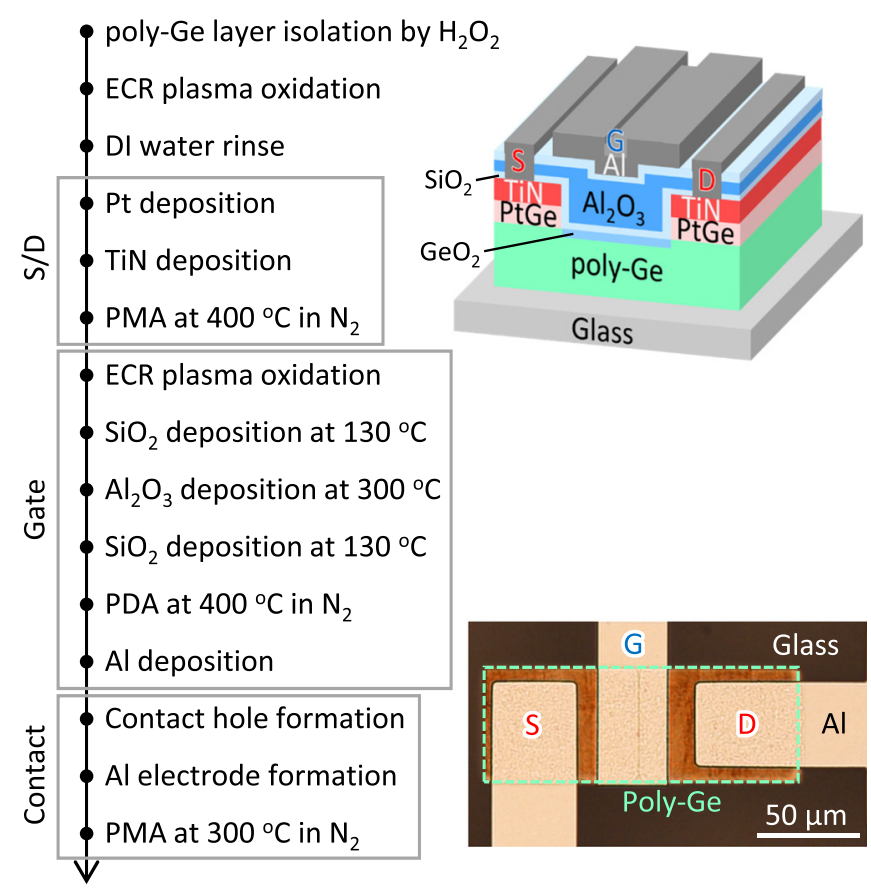

FIG. 2. Fabrication process flow (left) and schematic and an optical micrograph (right) of the p-channel SPC-Ge TFTs 
deposition at $300^{\circ} \mathrm{C}$. This $\mathrm{Al}_{2} \mathrm{O}_{3}$ film electrically isolates the sidewall of metal S/D and the gate electrode. To protect $\mathrm{Al}_{2} \mathrm{O}_{3}$ during gate electrode patterning, $\mathrm{SiO}_{2}$ ( $5 \mathrm{~nm}$ thick) was deposited by ECR plasma sputtering at $130^{\circ} \mathrm{C}$. Subsequently, postdeposition annealing (PDA) was performed at $400^{\circ} \mathrm{C}$ for $30 \mathrm{~min}$ in $\mathrm{N}_{2}$. As a gate electrode, $\mathrm{Al}$ (200 $\mathrm{nm}$ thick) was deposited using vacuum evaporation and patterned by wet etching. A contact hole was opened and an $\mathrm{Al}$ electrode ( $100 \mathrm{~nm}$ thick) was deposited using vacuum evaporation and patterned by a lift-off process. Finally, contact annealing was performed at $300^{\circ} \mathrm{C}$ for $30 \mathrm{~min}$ in $\mathrm{N}_{2}$. The channel width and length $(W / L)$ were 55 and $5-15 \mu \mathrm{m}$, respectively (Fig. 2). Here, all processes including SPC were conducted below $450{ }^{\circ} \mathrm{C}$.

We estimated $\mu_{\mathrm{FE}}$ of the TFTs using the following equation:

$$
\mu_{F E}=g_{m} \frac{L}{W} \frac{1}{C_{o x} V_{D}},
$$

where transconductance $g_{\mathrm{m}}$ was obtained from the drain current $\left(I_{\mathrm{D}}\right)$-gate voltage $\left(V_{\mathrm{G}}\right)$ characteristics. The gate oxide capacitance $C_{\mathrm{ox}}$, determined from the capacitance-voltage characteristics of the MOS capacitor with the same gate stack fabricated on the bulk Ge substrate, was $0.20 \mu \mathrm{F} / \mathrm{cm}^{2}$ corresponding to the equivalent oxide thickness of $17.7 \mathrm{~nm}$. The drain voltage $V_{\mathrm{D}}$ was fixed at $-0.1 \mathrm{~V}$. Figures $3(\mathrm{a})-3(\mathrm{~d})$ show that the $I_{\mathrm{D}}-V_{\mathrm{G}}$ and $\mu_{\mathrm{FE}}$ characteristics vary significantly with $t_{\mathrm{i}}$. Figure $3(\mathrm{e})$ shows on current $I_{\mathrm{on}}$, off current $I_{\mathrm{off}}$, and on/off current ratio $I_{\mathrm{on}} / I_{\text {off }}$ estimated from the $I_{\mathrm{D}}-V_{\mathrm{G}}$ characteristics. $I_{\text {on }}$ increases with increasing $t_{\mathrm{i}}$, which reflects $\mu_{\mathrm{Hall}}$ [Fig. $1(\mathrm{~g})$ ] and exhibits high values $\left(>10^{-4} \mathrm{~A}\right)$ at $t_{\mathrm{i}} \geq 100 \mathrm{~nm}$. $I_{\text {off }}$ is determined by the relationship between $t_{\mathrm{i}}$ and the maximum depletion layer width $d_{\max }$, which can be estimated from $p$. For example, the $d_{\max }$ of Ge when $p=3 \times 10^{17} \mathrm{~cm}^{-3}$ is approximately $54 \mathrm{~nm}$, assuming that the dielectric constant and intrinsic carrier concentration of Ge are 16 and $2.4 \times 10^{13} \mathrm{~cm}^{-3}$ at room temperature, respectively. From the relationship between $d_{\max }$ and $t_{\mathrm{i}}$, we found that the lower $t_{\mathrm{i}}$ provides the higher occupation of the depletion layer in the whole SPC-Ge layer, which decreases $I_{\text {off }}\left[\right.$ Fig. 3(e)]. Reflecting $I_{\text {on }}$ and $I_{\text {off }} I_{\text {on }} / I_{\text {off }}$ reaches the maximum at $t_{\mathrm{i}}=50 \mathrm{~nm}$. Figure $3(\mathrm{f})$ shows that $\mu_{\mathrm{FE}}$ is consistent with the trend of $\mu_{\mathrm{Hall}}$ reflecting the properties of SPC$\mathrm{Ge}$, while $\mu_{\mathrm{FE}}$ is much lower than $\mu_{\mathrm{Hall}}$. This is likely because not only carrier scattering at the MOS interface but also large $I_{\text {off }}$ causes underestimation of $g_{\mathrm{m}}$ and $\mu_{\mathrm{FE}}$. To overcome this problem, a-Ge layer compatible with high $\mu_{\text {Hall }}$ (corresponding high $I_{\mathrm{on}}$ ) and a thin film (corresponding low $I_{\text {off }}$ ) is desirable.

Considering the aspect ratio of the grain size and film thickness for $t_{\mathrm{i}} \geq 50 \mathrm{~nm}$, the grain size is constant in the depth direction for each $t_{\mathrm{i}}$. The influence of the carrier scattering near the $\mathrm{Ge} / \mathrm{SiO}_{2}$ interface should be identical at the same thickness. Therefore, for achieving high $\mu_{\text {Hall }}$ with a thin film, we thinned the $t_{\mathrm{i}}=100 \mathrm{~nm}$ sample, which has the largest grain size [Fig. 1(f)], using chemical-mechanical polishing (CMP). Figure 4(a) shows that $p$ is constant in the depth direction. According to $d_{\max }$ estimated from $p$, full depletion in SPC-Ge is obtained when the thinned film thickness $t_{\mathrm{CMP}}$ is below $55 \mathrm{~nm}$. Conversely, $\mu_{\text {Hall }}$ decreases significantly for $t_{\mathrm{CMP}}<50 \mathrm{~nm}$ [Fig. 4(b)], likely reflecting carrier scattering at the $\mathrm{Ge} / \mathrm{SiO}_{2}$ interface. ${ }^{15,30}$ Therefore, we determined that $t_{\mathrm{CMP}}=55 \mathrm{~nm}$ is almost optimal for achieving both high $\mu_{\mathrm{FE}}$ and $I_{\mathrm{on}} / I_{\text {off. The }} t_{\mathrm{CMP}}=55 \mathrm{~nm}$ sample showed a smooth surface [Fig. 4(b), root mean square value: $1.3 \mathrm{~nm}$ ], and the same grain size as before CMP. This sample was processed into TFTs by the procedure shown in Fig. 2. Figure 4(c) shows the
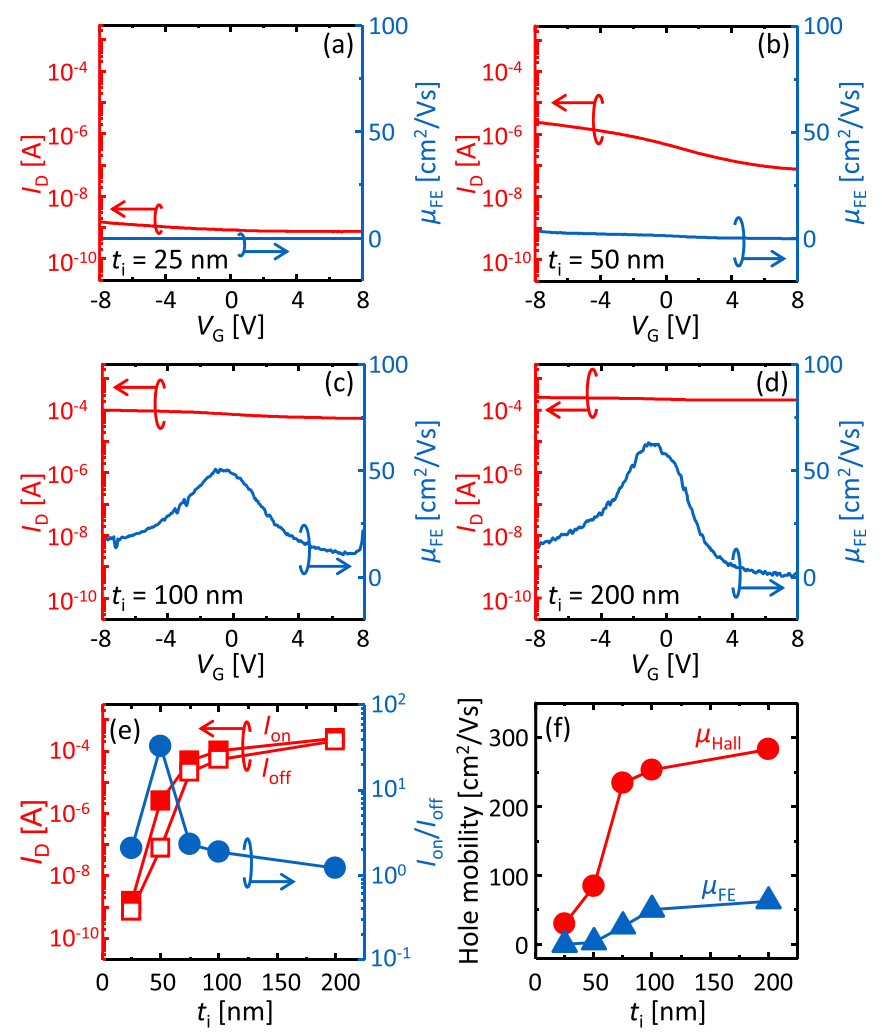

FIG. 3. Electrical properties of $p$-channel SPC-Ge TFTs with various initial film thickness $t_{i}$ values, where channel length $L=10 \mu \mathrm{m}$. (a)-(d) $I_{D}-V_{G}$ and field-effect mobility $\mu_{\mathrm{FE}}$ characteristics at $V_{\mathrm{D}}=-0.1 \mathrm{~V}$. (e) On current $I_{\mathrm{on}}$, off current $I_{\text {off, }}$ and on/off current ratio $I_{\mathrm{on}} / I_{\text {off }}$ and (f) $\mu_{\mathrm{FE}}$ and Hall effect mobility $\mu_{\text {Hall }}$ as a function of $t_{\mathrm{j}}$.

typical p-channel transistor operation, i.e., $I_{\mathrm{D}}$ increases with increasing $V_{\mathrm{G}}$. Figure $4(\mathrm{~d})$ shows high $I_{\mathrm{on}} / I_{\mathrm{off}}\left(10^{2}\right)$ and $\mu_{\mathrm{FE}}\left(170 \mathrm{~cm}^{2} / \mathrm{Vs}\right)$ because of both the high $I_{\text {on }}$ due to the high $\mu_{\text {Hall }}$ and low $I_{\text {off }}$ due to the thin thickness $(55 \mathrm{~nm})$ in SPC-Ge. The current TFT performance is the highest among simple poly-Ge TFTs formed on glass and will be further improved by nanofabrication processes such as miniaturization of the channel region and multigate structure. For TFTs fabricated on polycrystalline semiconductors, their variability is also important. Specifically, it is more sensitive in the case of devices with a channel length comparable to the grain size like this study. The cumulative distribution of measured $\mu_{\mathrm{FE}} \mathrm{S}$ for different channel lengths $(5,10$, and $15 \mu \mathrm{m})$ in the same substrate is shown in Fig. 4(e). The experimental data show a clear increase in variability as the channel length decreases, which is in good agreement with the trends reported in Ref. 34 from both the theoretical and experimental viewpoints. Device variability will also be improved by device design optimization for the corresponding grain size.

In conclusion, we studied the relationship between film properties (thickness, $\mu_{\mathrm{Hall}}$, and $p$ ) and TFT characteristics ( $\mu_{\mathrm{FE}}$ and $\left.I_{\mathrm{on}} / I_{\mathrm{off}}\right)$ using high-mobility poly-Ge layers formed on glass by SPC. Thicker $t_{\mathrm{i}}$ enabled higher $\mu_{\mathrm{FE}}$ because of higher $\mu_{\mathrm{Hall}}$, while it enabled larger $I_{\mathrm{off}}$, which led to poor $I_{\mathrm{on}} / I_{\mathrm{off}}$. These results indicated that both high $\mu_{\mathrm{Hall}}$ and thin $t_{\mathrm{i}}$ are essential to achieve both high $\mu_{\mathrm{FE}}$ and $I_{\mathrm{on}} / I_{\mathrm{off}}$. By thinning a $100-\mathrm{nm}$ thick Ge layer with a large grain size $(3.7 \mu \mathrm{m})$, we achieved a high $\mu_{\mathrm{Hall}}\left(190 \mathrm{~cm}^{2} / \mathrm{Vs}\right)$ at a thin film $(55 \mathrm{~nm})$ that is almost 

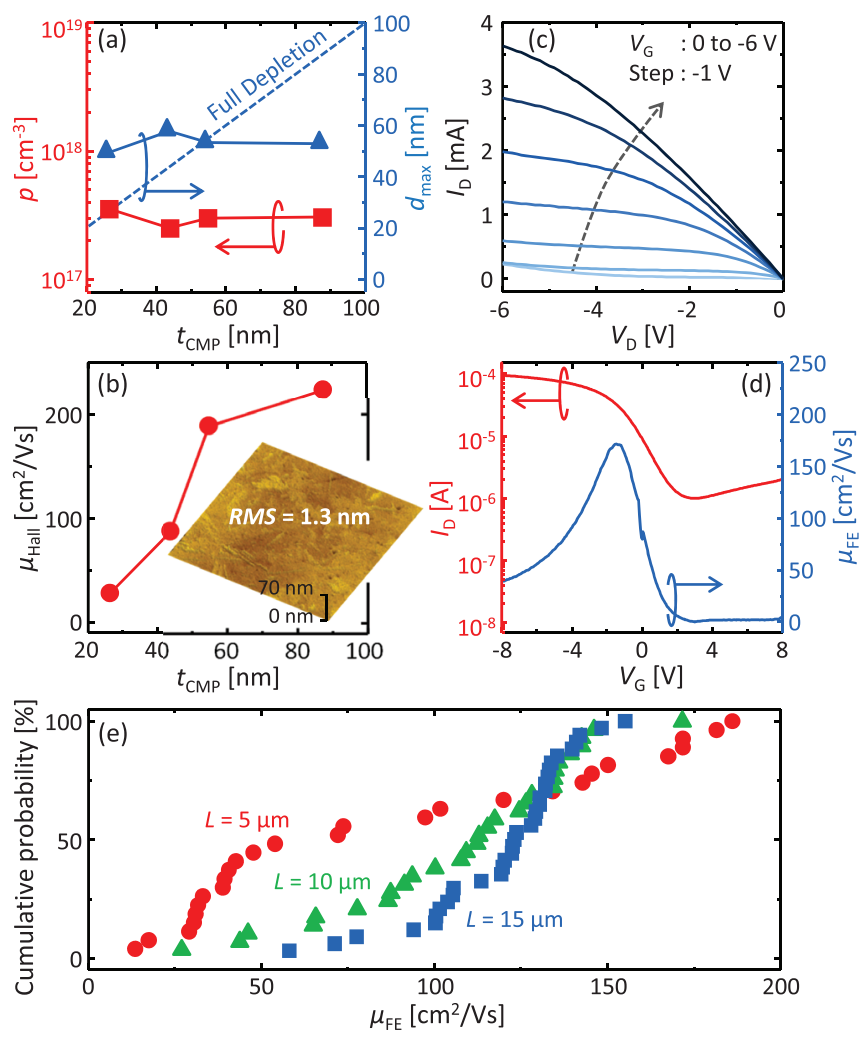

FIG. 4. (a) and (b) "In-depth" profiles of electrical properties for SPC-Ge layers for initial film thickness $t_{\mathrm{i}}=100 \mathrm{~nm}$. (a) Hole concentration $p$ and maximum depletion layer width $d_{\max }$ estimated from $p$ as a function of film thickness $t_{\text {CMP }}$ thinned by using CMP. The dotted line shows the line when the Ge layer is fully depleted. (b) Hall hole mobility $\mu_{\text {Hall }}$ as a function of $t_{\text {CMP. }}$. The inset in (b) shows the atomic force micrograph of SPC-Ge thinned to $t_{\mathrm{CMP}}=55 \mathrm{~nm}$ with a scan region of $10 \times 10 \mu \mathrm{m}^{2}$. (c) $I_{D}-V_{D}$ characteristics and (d) $I_{D}-V_{G}$ characteristics and field-effect mobility $\mu_{\mathrm{FE}}$ at $V_{\mathrm{D}}=-0.1 \mathrm{~V}$ for the SPC-Ge TFT thinned to $t_{\mathrm{CMP}}=55 \mathrm{~nm}$, where channel length $L=10 \mu \mathrm{m}$. (e) Cumulative distribution of $\mu_{\mathrm{FE}}$ with different $L$ values.

thin enough to fully deplete the channel. The TFT using this Ge layer exhibited high $\mu_{\mathrm{FE}}\left(170 \mathrm{~cm}^{2} / \mathrm{Vs}\right)$ and $I_{\mathrm{on}} / I_{\mathrm{off}}\left(10^{2}\right)$. The $\mu_{\mathrm{FE}}$ value is the highest among low-temperature $\left(<500^{\circ} \mathrm{C}\right)$ polycrystalline Ge TFTs without minimizing the channel region $(<1 \mu \mathrm{m})$. The findings in the present study will contribute to the development of high-performance poly-Ge-TFTs that exceed Si-MOSFETs and lead to advanced threedimensional LSIs and multifunctional mobile terminals.

This work was financially supported by a Grant-in-Aid for JSPS Research Fellows (No. 17J00544), the Murata Science Foundation, and JST PRESTO (No. JPMJPR17R7). The authors are grateful to Dr. T. Sakurai (University of Tsukuba) for assistance with the Hall effect measurements. EBSD measurements were conducted at the International Center for Young Scientists at NIMS.

\section{REFERENCES}

${ }^{1}$ A. Nayfeh, C. O. Chui, T. Yonehara, and K. C. Saraswat, IEEE Electron Device Lett. 26, 311 (2005).
${ }^{2}$ D. P. Brunco, B. De Jaeger, G. Eneman, J. Mitard, G. Hellings, A. Satta, V. Terzieva, L. Souriau, F. E. Leys, G. Pourtois, M. Houssa, G. Winderickx, E. Vrancken, S. Sioncke, K. Opsomer, G. Nicholas, M. Caymax, A. Stesmans, J. Van Steenbergen, P. W. Mertens, M. Meuris, and M. M. Heyns, J. Electrochem. Soc. 155, H552 (2008).

${ }^{3}$ R. Pillarisetty, Nature 479, 324 (2011).

${ }^{4}$ K. Yamamoto, T. Sada, D. Wang, and H. Nakashima, Appl. Phys. Lett. 103, 122106 (2013).

${ }^{5}$ S. Takagi, R. Zhang, J. Suh, S.-H. Kim, M. Yokoyama, K. Nishi, and M. Takenaka, Jpn. J. Appl. Phys. Part 1 54, 06FA01 (2015).

${ }^{6}$ A. Toriumi and T. Nishimura, Jpn. J. Appl. Phys. Part 1 57, 010101 (2018).

${ }^{7}$ G. Taraschi, A. J. Pitera, and E. A. Fitzgerald, Solid-State Electron. 48, 1297 (2004).

${ }^{8}$ Y. Moriyama, K. Ikeda, Y. Kamimuta, M. Oda, T. Irisawa, Y. Nakamura, A. Sakai, and T. Tezuka, Solid-State Electron. 83, 42 (2013).

${ }^{9}$ K. Yu, F. Yang, H. Cong, L. Zhou, Q. Liu, L. Zhang, B. Cheng, C. Xue, Y. Zuo, and C. Li, J. Alloys Compd. 750, 182 (2018).

${ }^{10}$ T. Maeda, K. Ikeda, S. Nakaharai, T. Tezuka, N. Sugiyama, Y. Moriyama, and S. Takagi, Thin Solid Films 508, 346 (2006).

${ }^{11}$ J. Feng, G. Thareja, M. Kobayashi, S. Chen, A. Poon, Y. Bai, P. B. Griffin, S. S. Wong, Y. Nishi, and J. D. Plummer, IEEE Electron Device Lett. 29, 805 (2008).

${ }^{12}$ S. Hu, P. W. Leu, A. F. Marshall, and P. C. McIntyre, Nat. Nanotechnol. 4, 649 (2009).

${ }^{13}$ K. Toko, Y. Ohta, T. Tanaka, T. Sadoh, and M. Miyao, Appl. Phys. Lett. 99, 032103 (2011).

${ }^{14}$ T. Hosoi, Y. Suzuki, T. Shimura, and H. Watanabe, Appl. Phys. Lett. 105, 173502 (2014).

${ }^{15}$ K. Usuda, Y. Kamata, Y. Kamimuta, T. Mori, M. Koike, and T. Tezuka, Appl. Phys. Express 7, 056501 (2014).

${ }^{16}$ Y. Kamata, M. Koike, E. Kurosawa, M. Kurosawa, H. Ota, O. Nakatsuka, S. Zaima, and T. Tezuka, Appl. Phys. Express 7, 121302 (2014).

${ }^{17}$ T. Sadoh, H. Kamizuru, A. Kenjo, and M. Miyao, Appl. Phys. Lett. 89, 192114 (2006).

${ }^{18}$ K. Toko, I. Nakao, T. Sadoh, T. Noguchi, and M. Miyao, Solid-State Electron. 53, 1159 (2009).

${ }^{19}$ A. Hara, Y. Nishimura, and H. Ohsawa, Jpn. J. Appl. Phys. Part 1 56, 03 BB01 (2017).

${ }^{20}$ S. Kabuyanagi, T. Nishimura, K. Nagashio, and A. Toriumi, Thin Solid Films 557, 334 (2014).

${ }^{21}$ H. A. Kasirajan, W.-H. Huang, M.-H. Kao, H.-H. Wang, J.-M. Shieh, F.-M. Pan, and C.-H. Shen, Appl. Phys. Express 11, 101305 (2018).

${ }^{22}$ M. Asadirad, Y. Gao, P. Dutta, S. Shervin, S. Sun, S. Ravipati, S. H. Kim, Y. Yao, K. H. Lee, A. P. Litvinchuk, V. Selvamanickam, and J.-H. Ryou, Adv. Electron. Mater. 2, 1600041 (2016).

${ }^{23}$ B. Hekmatshoar, S. Mohajerzadeh, D. Shahrjerdi, and M. D. Robertson, Appl. Phys. Lett. 85, 1054 (2004).

${ }^{24}$ K. Toko, R. Numata, N. Oya, N. Fukata, N. Usami, and T. Suemasu, Appl. Phys. Lett. 104, 22106 (2014).

${ }^{25}$ K. Kasahara, Y. Nagatomi, K. Yamamoto, H. Higashi, M. Nakano, S. Yamada, D. Wang, H. Nakashima, and K. Hamaya, Appl. Phys. Lett. 107, 142102 (2015).

${ }^{26}$ T. Suzuki, B. M. Joseph, M. Fukai, M. Kamiko, and K. Kyuno, Appl. Phys. Express 10, 095502 (2017).

${ }^{27}$ H. Higashi, K. Kudo, K. Yamamoto, S. Yamada, T. Kanashima, I. Tsunoda, H. Nakashima, and K. Hamaya, J. Appl. Phys. 123, 215704 (2018).

${ }^{28}$ H. Haesslein, R. Sielemann, and C. Zistl, Phys. Rev. Lett. 80, 2626 (1998).

${ }^{29} \mathrm{~K}$. Toko, R. Yoshimine, K. Moto, and T. Suemasu, Sci. Rep. 7, 16981 (2017).

${ }^{30}$ R. Yoshimine, K. Moto, T. Suemasu, and K. Toko, Appl. Phys. Express 11, 031302 (2018).

${ }^{31}$ T. Imajo, K. Moto, R. Yoshimine, T. Suemasu, and K. Toko, Appl. Phys. Express 12, 015508 (2019).

${ }^{32}$ Y. Kimura, M. Kishi, and T. Katoda, J. Appl. Phys. 86, 2278 (1999).

${ }^{33}$ A. Dimoulas, A. Toriumi, and S. E. Mohney, MRS Bull. 34, 522 (2009).

${ }^{34}$ A. W. Wang and K. C. Saraswat, IEEE Trans. Electron Devices 47, 1035 (2000). 